"This is the peer reviewed version of the following article: Arias, Francisco J., De las Heras, Salvador. Hydrokinetic energy conversión using compliant surfaces.

“International journal of energy research", 2019, vol. 43, núm. 9, p. 4427-4435, which has been published in final form at [https://doi.org/10.1002/er.4568]. This article may be used for non-commercial purposes in accordance with Wiley Terms and Conditions for Use of Self-Archived Versions." 


\title{
Hydrokinetic Energy Conversion Using Compliant Surfaces
}

\author{
Francisco J. Arias ${ }^{a}$, Salvador De Las Heras ${ }^{a}$ \\ aDepartment of Fluid Mechanics, University of Catalonia, \\ ESEIAAT C/ Colom 11, 08222 Barcelona, Spain \\ francisco.javier.arias@upc.edu
}

\section{Summary}

In this work, consideration is given for a novel technology based on the use of compliant surfaces (membranes) for hydrokinetic energy conversion (HEC) from residual waters where the use of traditional turbines is either not possible or strongly limited. Here, residual waters are understood as waters with a small total pressure difference relative to the surrounding environment that are not suitable to be turbined. Such waters encompass not only domestic or industrial waste water before being discharged into the sewer but also typical flows found in river waterways of small depth or even post-turbined water. The proposed inexpensive technology of membranes for hydrokinetic energy conversion is driven only by the dynamic pressure of the total pressure and does not need static pressure to run as is the case of turbines. In summary, the proposed technology is based on the deliberated bifurcation of residual water into two streams separated by a compliant surface or membrane that equalizes the static pressure in both streams and also generates a relative velocity between them. As a result, Taylor instabilities translate into the oscillatory motion of the membrane, which can be transformed into output power. Utilizing linearized flow theory, an analytical expression for the extractable density power is derived. Experiments are carried out for a rectangular membrane, and the data obtained are quantitatively and qualitatively in good agreement with the theoretical model, where it is found that for typical residual water with a velocity of approximately $1.7 \mathrm{~m} / \mathrm{s}$, the output power density is on the order of $30 \mathrm{~mW} / \mathrm{cm}^{2}$ based on the area of the membrane. Additional research and development is required to arrive at a reliable practical and commercial design.

Keywords. Energy harvesting; hydrokinetic energy conversion (HEC); residual waters

\section{INTRODUCTION}

Energy harvesting or energy scavenging is the process by which wasted energy can be captured and stored from different types of waste sources and technologies and used for small, wireless autonomous devices, such as those used in wearable electronics and wireless sensor networks or nanotechnology with typical inputs from $1 \mu \mathrm{W}$ up to a few $\mathrm{mW}$. Energy harvesting can be performed from different sources, such as thermal energy ${ }^{1-3}$; wind energy ${ }^{4,5}$; salinity gradients $^{6}$; piezoelectric energy ${ }^{7}$; triboelectricity ${ }^{8}$; vibrational energy ${ }^{9}$; and hydrokinetic energy 10 including wave energy conversion ${ }^{11}$, just to name a few.

In regards to hydrokinetic power, which is electricity generated from water in motion, the current harvesting technology is almost entirely based on the use of water turbines, which 
transform the water's energy into mechanical energy, similar to the way wind turbines transform wind energy, and although they can vary in size and design, they are generally classified into three main types as vertical-axis hydrokinetic turbines ${ }^{12}$, horizontal-axis hydrokinetic turbines ${ }^{13}$, and oscillating-foil hydrokinetic turbines ${ }^{14,15}$. For readers interested in an up-to date review on hydrokinetic energy conversion, see ${ }^{10}$.

Nevertheless, because turbines require a minimum pressure drop with the surrounding environment as well as a minimum cross-sectional area (generally limited by the width of the blades), they are therefore often not a feasible solution for application in residual waters which frequently move through narrow channels and generally move at low total pressure (dynamic plus static); in addition, they frequently transport solid material that may jeopardize the performance of the turbine. The objective of this work was to analyse a possible novel hydrokinetic energy harvester based on the use of compliant surfaces or membranes that are more suitable for residual waters, i.e., water flows with a low total pressure relative to the surrounding environment.

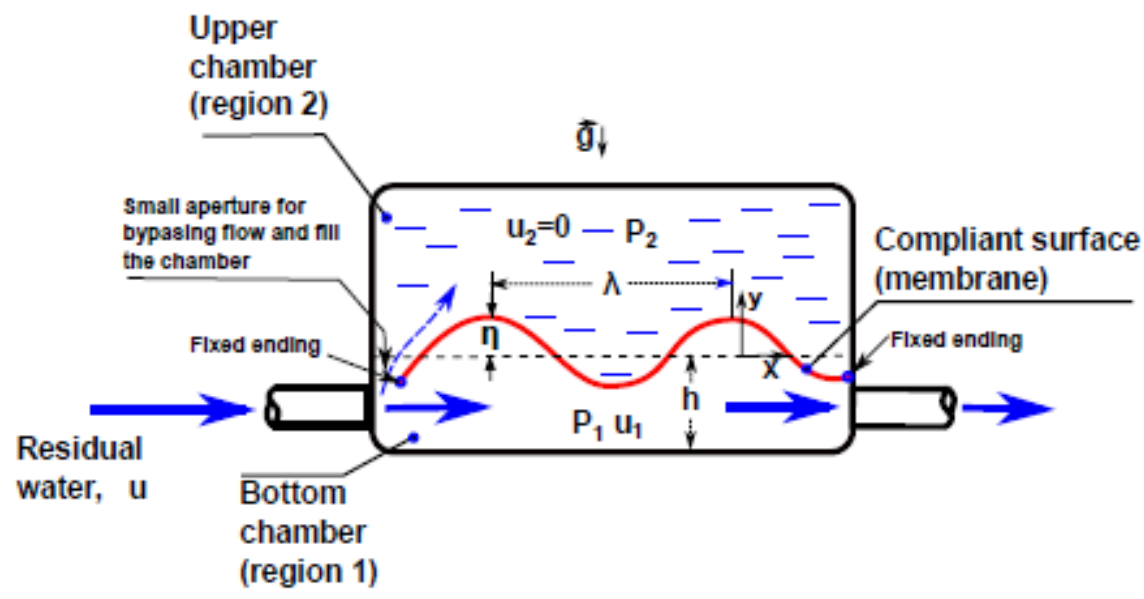

FIGURE 1 Physical sketch of the hydrokinetic energy harvesting method by using a compliant surface.

\section{STATEMENT OF THE CORE IDEA}

To begin with, let us consider the system schematically depicted in Figure 1, where a stream of residual water (i.e., with a low differential pressure with the surrounding environment but with a certain velocity) is flowing with velocity $u$ into a certain cavity in the $x$ direction in which the water is deliberately bifurcated into two chambers, the upper and bottom chamber, which are separated by a compliant surface (membrane) at a distance $h$ from the bottom and fastened at the ends in such a way that it can move freely. We denote the vertical displacement of the membrane from the equilibrium position in the plane $y=0$ by $\eta$. 


\subsection{Equations of Motion}

First, we consider the motion of the membrane subject to the following simplifying assumptions: (1) The displacements or transverse vibrations of the compliant surface are small (very small compared to the wavelength) so that the equations of a stretched elastic membrane are applicable; therefore, both the compliant surface and fluid behave according to linearized flow theory; and (2) the membrane is wide enough that edge effects are negligible. This simplified model seems justified in view of the uncertainties and complexity of the problem and taking into account that for now, our motivation is to develop an analytical model able to provide reasonable estimates of the theoretical limit of the extractable energy.

A complete derivation of linearized hydrodynamic theory is somewhat complex, and the readier is referred to the classic texts by ${ }^{16,17}$ for the derivation.

In general, according to Taylor's analysis within the linearized theory (assuming potential flow), the shape of the interface disturbance introduced into the first-order perturbation analysis for a travelling wave is given by

$$
\eta=\eta_{e} e^{\text {tinxthet }}
$$

where $\eta$ is the amplitude of the membrane wave at time $t ; \eta_{\circ}$ is the small-disturbance amplitude; $m=\frac{2 \pi}{\lambda}$ is the wavenumber with wavslongth $\lambda ; \omega=\frac{i \pi}{\tau}$ is the wave frequency and $T$ is the wave period.

The hydrodynamic equations for each of the two fluid regions are ${ }^{17}$

$$
\begin{aligned}
& \frac{\partial_{v}}{\partial y}+\frac{g_{w}}{g_{x}}=0 \\
& \frac{\partial_{w}}{\partial_{x}}+U \frac{\partial w}{\partial x}=-\frac{1}{\theta} \frac{\partial_{x}}{\partial x} \\
& \frac{\partial_{v}}{\partial_{v}}+U \frac{\partial_{v}}{\partial_{w}}=-\frac{1}{\partial} \frac{\partial_{y}}{\partial x}
\end{aligned}
$$

subject to the boundary conditions

and

$$
v_{2}(x, m, t)=0
$$

$$
v_{1}\left(x_{p}-k_{p}, t\right)=0
$$

where $h$ is the depth of the cavity as defined in Figure 1.

For a potential flow, the velocity can be expressed as a function of the gradient of a potential function, and then Eqs. (2)-(4) yield 


$$
\begin{gathered}
u=U-\frac{\partial \phi}{\partial x} \\
v=-\frac{\partial \phi}{\partial y}
\end{gathered}
$$

and

$$
p=\rho \frac{\partial \phi}{g t}+\rho U \frac{Q \phi}{\partial x}-\rho Q y
$$

where $\phi$ is the velocity potential function, which for an incompressible fluid gives

$$
\nabla^{2} \phi-0
$$

Taking into account Eq. (8) and the boundary conditions Eq. (5) and Eq. (6), we have

$$
\frac{\partial \phi}{8 x}(x, \infty, t)=0
$$

and

$$
\frac{\partial \phi h}{8 x}\left(x_{z}-k_{v} t\right)=0
$$

The potential function satisfying Eq. (10)-Eq. (12) for the bottom region is given by

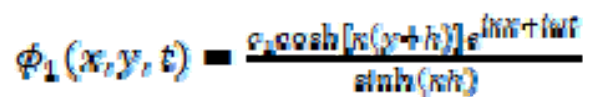

and for the upper fluid

$$
\Phi_{2}\left(x, y_{t} t\right)=\varepsilon_{2} e^{-n y_{e} t n x+w s}
$$

where $c_{1}$ and $c_{2}$ are constants to be determined by the boundary conditions. Taking into account that the velocity at the membrane is made up of the velocity of the membrane itself and the velocity of the fluid relative to the membrane, for $y=\eta(x, t)$

$$
\begin{gathered}
v_{1}\left(x_{v} \eta_{v} t\right)=\frac{g_{n}}{\partial_{v}}+U_{1} \frac{g_{n}}{\partial x} \\
v_{2}\left(x_{v} \eta \eta_{v} t\right)=\frac{g_{n}}{\partial t}
\end{gathered}
$$

and then

$$
C_{1}=-\left[\frac{m_{\gamma} \omega}{\kappa}+t n_{e} I_{1}\right]
$$


and

$$
\varphi_{2}=\frac{m_{0} L}{n}
$$

considering small oscillations, $\eta \approx 0$, and $(\eta+h) \approx h$. Finally, the pressure condition across the membrane, Eq. (9), yields

$$
p_{1}-p_{2}=-\left[\left(t \omega+t \kappa U_{1}\right)\left(\frac{m_{3} \omega}{\kappa}+t \eta_{\Omega} U_{1}\right) \operatorname{coth}(\kappa h)+(t \omega)\left(\frac{m_{\beta} \omega}{\kappa}\right)\right] \rho e^{t \kappa x+t \omega t}
$$

Because the typical wavelengths for our harvester are very small (around millimetres), a valid simplifying assumption is that $k \leqslant k$; therefore,

$$
\operatorname{coth}(k h)=1
$$

Thus, Eq. (19) simplifies as

$$
P_{1}-p_{2} \approx-\left[2 \omega U_{1}+\kappa U_{1}^{0}\right] \eta_{\sigma} R e^{\hbar \times+\hbar \omega t}
$$

As we will see below, the natural oscillation frequency for a membrane stretched could be approximately $10 \mathrm{Hertz}$, and on the other hand, $\approx \approx 2 \pi \times 10^{3} \mathrm{~m}^{-1}$, and then it is allowable to assume $\kappa U_{1}^{2} \gg 2 \omega U$, and then Eq. (21) further simplifies as

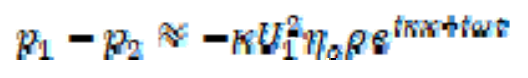

\subsection{Membrane wavelength}

Eq. (22) gives the pressure condition across the membrane. This pressure also must be equal to the pressure by the deflection of the membrane. If one considers that the vibrations of the compliant surface are very small (assumption (1)), then the equations of a stretched elastic membrane are applicable and may be roughly approximated with a certain surface tension $\Gamma$. The pressure resulting from the curvature of the interface $\eta$ is given by ${ }^{17}$

$$
p_{1}-v_{2}=2 \Gamma \frac{\partial^{2} n}{d x^{2}}
$$




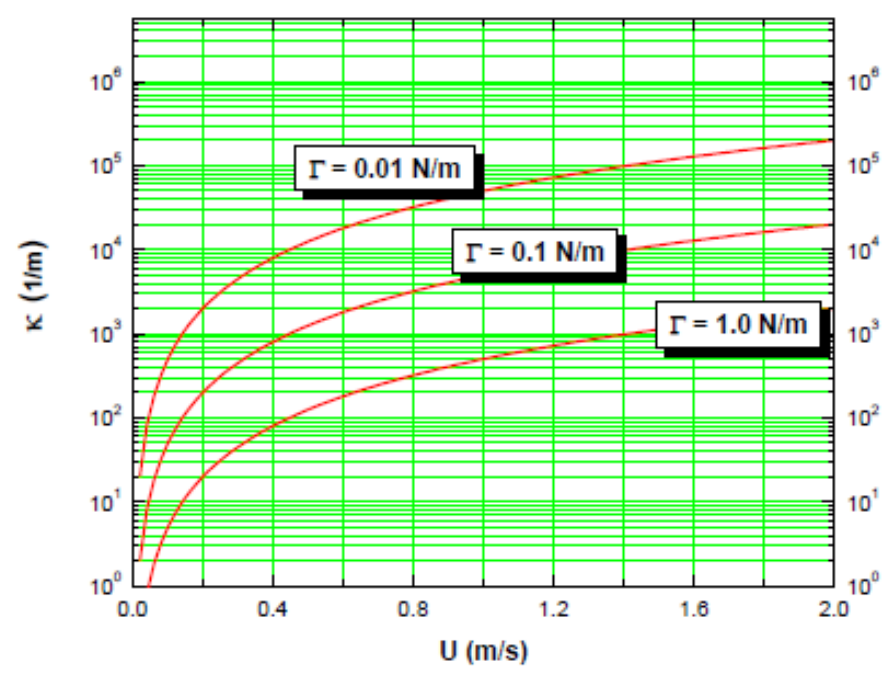

FIGURE 2 Plot of $\kappa$ as a function of $U$ from Eq. (25) for water and several values of the membrane tension

which considering Eq. (1) becomes

$$
v_{1}-p_{2}=-2 \Gamma \psi_{e} \kappa^{2} e^{k w+t \omega t}
$$

Thus, by equating Eq. (24) with Eq. (22), we obtain

$$
K=\frac{V_{R}^{R} R}{2 R^{2}}
$$

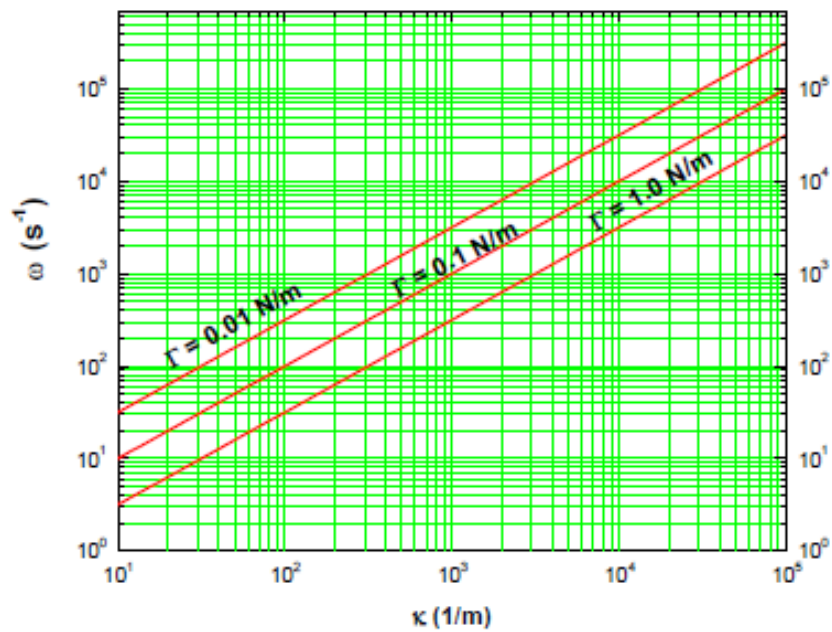

FIGURE 3 Plot of $\omega$ as a function of $\kappa$ from Eq. (27) for water and several values of the membrane tension with a membrane density of $\rho_{m}=0.1 \mathrm{~kg} / \mathrm{m}^{2}$. 


\subsection{Membrane natural frequency}

For a rectangular membrane, we have the following dynamic equation ${ }^{18}$

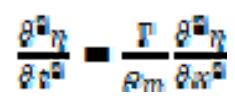

where $\rho_{m}$ is the mass of the undeflected membrane per unit area. The solution of Eq. (26) gives us the natural oscillation frequency for a stretched membrane as ${ }^{18}$

$$
\omega-\pi \sqrt{\frac{r}{\rho_{m}}}
$$

\subsection{Discussion}

Figure 1 shows the curve predicted by Eq. (25) considering membrane tensions of $\Gamma=1.0 \mathrm{~N} / \mathrm{m}, \Gamma=0.1 \mathrm{~N} / \mathrm{m}$, and $\Gamma=0.01 \mathrm{~N} / \mathrm{m}$. It is seen that for fluid flows approximately 1 $\mathrm{m} / \mathrm{s}$ to $2 \mathrm{~m} / \mathrm{s}$ as can be found in residual waters, the wavelength can be around $\lambda=2.5 \times 10^{-5}$ $\mathrm{m}$ to $\lambda=2.5 \times 10^{-6} \mathrm{~m}$ for tensions of $\Gamma=1.0 \mathrm{~N} / \mathrm{m}$ to $\Gamma=0.01 \mathrm{~N} / \mathrm{m}$, respectively. Figure 3 shows the curve predicted by Eq. (27) for a membrane with a membrane density of $p_{m}=0.05$ $\mathrm{kg} / \mathrm{m}^{2}$. It is seen that typical frequencies of approximately $10 \mathrm{~Hz}$ to $100 \mathrm{~Hz}$ are characteristic.

\subsection{Extractable energy}

The differential pressure vertical component force $f_{y}$ of the surface is given by

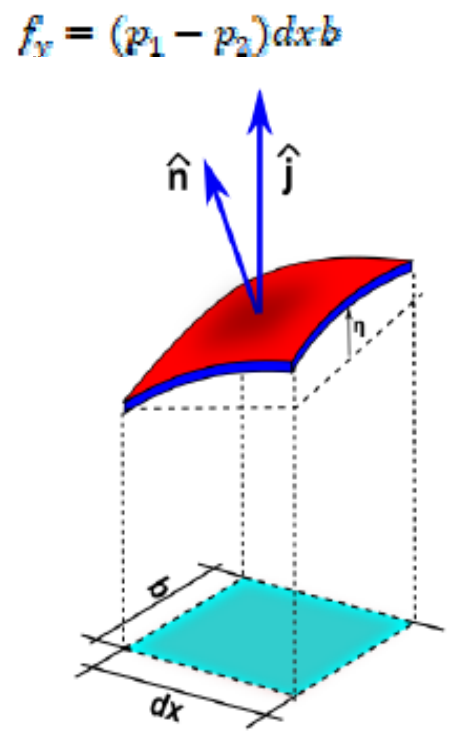

FIGURE 4 Surface element of the vibrating membrane. 
and the power at a given time and location is calculated considering the velocity of the membrane

$$
\omega(x, t)=f_{y} \frac{\partial \eta}{g_{t}}
$$

The power of the rectangular membrane is obtained by integrating Eq. (29) over the membrane area. Thus, taking into account Eq. (1), Eq. (22) and Eq. (29) and considering the $x$ origin at the centre of the membrane, the power for a membrane with, unstretched length $l$ yields

$$
w(t)=\frac{b E_{k}^{2} \pi \rho x^{2} \omega}{2} \int_{-i / 2}^{i / 2} \sin (2 \kappa x+2 \omega t) d x
$$

where a specific travelling wave $e^{k n+t \omega t}=\cos (k x+\omega t)$ is considered. The timeaveraged power over one cycle is obtained as

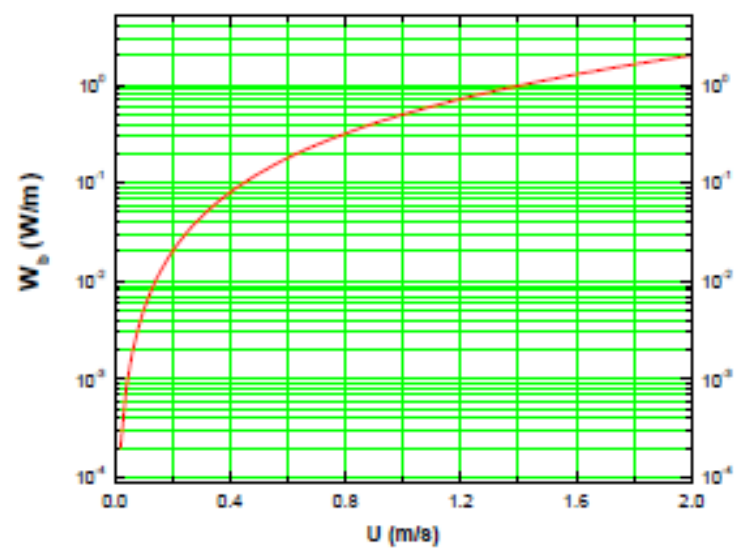

FIGURE 5 Plot of the power per membrane width $w_{b}$ as a function of water velocity $U$ from Eq. (34)

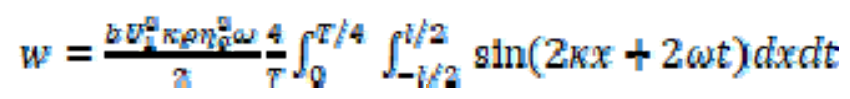

After integration of Eq. (29), one obtains

$$
w=\frac{2 b E^{2} \sin R^{2}}{7} \sin \alpha
$$

where it is considered that $\omega=\frac{2 \pi}{\tau}$, and the sign is unimportant. The power by unit crest 
width of the compliant surface $w_{b 2}=\frac{w}{b}$ is

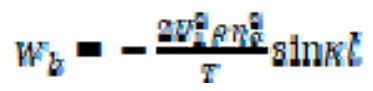

When $l \rightarrow 0$, it is a differential $l=d l$ and $\sin \kappa l \approx k d l$. Then, a power density per unit area may be defined as

$$
w_{s}=2 U_{1}^{2} \rho n_{\sigma}^{2} \frac{\kappa}{T}
$$

To obtain a first estimation of the maximum power predicted by Eq. (34), we assume some values of the parameters: $\rho=10^{3} \mathrm{~kg} / \mathrm{m}^{3}$ for water, a frequency of $10 \mathrm{~s}^{-1}$, and a plausible wave amplitude of $\eta_{\odot} \approx 0.5 \mathrm{~cm}$. The resulting curve is shown in Figure 5 .

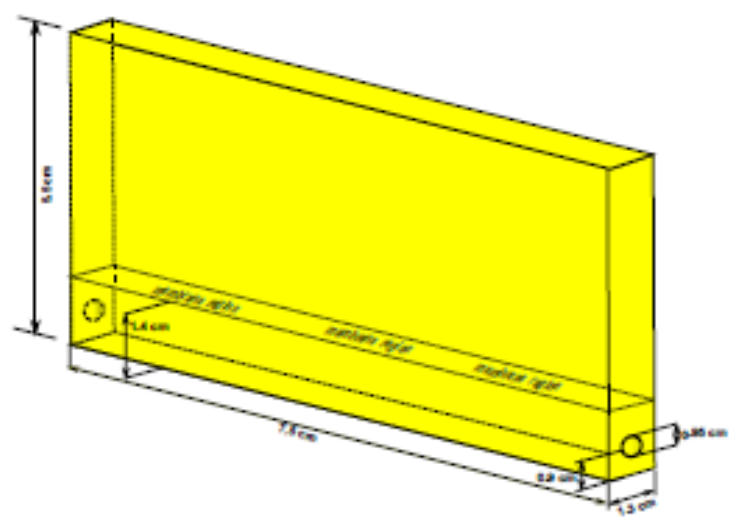

FIGURE 6 Sketch of the experimental setup

\section{EXPERIMENTAL VALIDATION}

Actual experimental investigations were carried out by using several cavities and configurations until the best design was attained. The experiment was performed in a rectangular narrow cavity $7.5 \mathrm{~cm}$ long, $5.5 \mathrm{~cm}$ high and $1.3 \mathrm{~cm}$ in width and with two perforations of $0.95 \mathrm{~cm}$ in diameter (for the entry and exit of water) cantered on the sides of the cavity $0.89 \mathrm{~cm}$ from the bottom. The membrane was located $1.4 \mathrm{~cm}$ from the bottom as depicted in Figure 6 . A stream of fresh water of density $\rho-1 \mathrm{~g} / \mathrm{cm}^{3}$ and kinematic viscosity $0.01 \mathrm{~cm}^{2} / \mathrm{s}$ from a domestic water intake with a 3.5 bar exit pressure and a temperature of 290 $\mathrm{K}$ was used for the experiment. The cavity was built with methacrylate for visualization. 


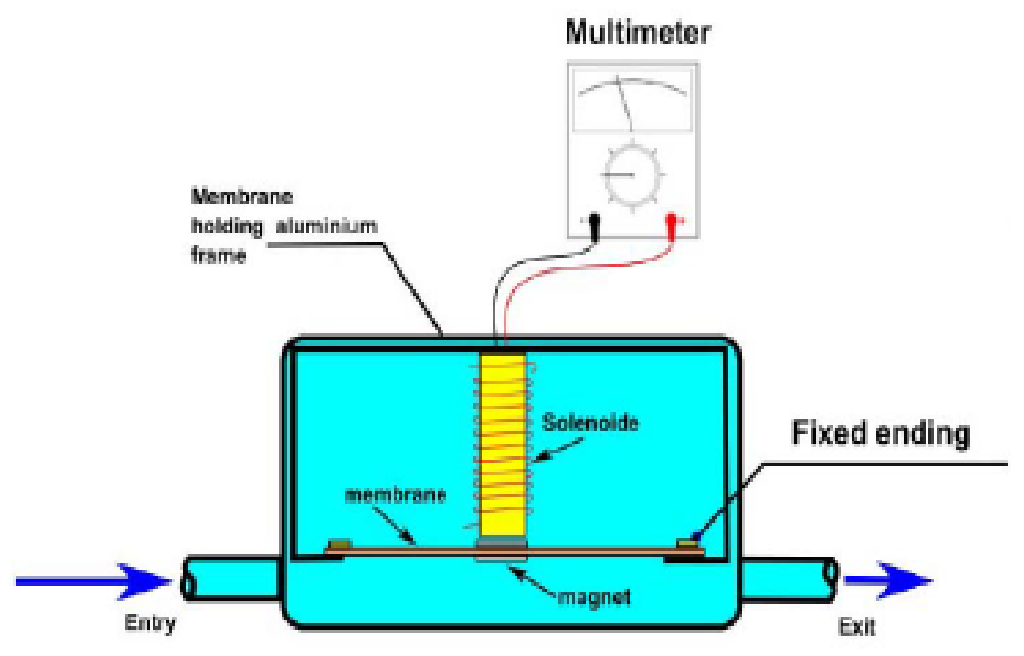

FIGURE 7 Sketch of the experimental setup.

The membrane was made of commercial rubber membrane that was $6 \mathrm{~cm}$ long, $1.2 \mathrm{~cm}$ in width and $1 \mathrm{~mm}$ in thickness and with a surface tension $5.5 \mathrm{~N} / \mathrm{m}$ for small displacements from its unstretched position. It was centred and positioned using an aluminium frame that fixed its ends but allowed a longitudinal gap of $\approx 0.05 \mathrm{~cm}$ for the free circulation of water to equalize the static pressure on both sides of the membrane and the oscillatory up and down motion. Although fixing the membrane at its ends implies the formation of standing waves rather than travelling waves as theoretically analysed previously, because of the small millimetric amplitudes, great deviations in the attainable power are not expected.

Finally, in order to extract the energy of the vibrating membrane, a linear inductance generator was chosen that simply consisted of a permanent magnet attached to the surface of the membrane and moved through a coil positioned vertically, as shown in Figure 7. The single coil was a conducting copper wire loop $2.5 \mathrm{~cm}$ long, with an external diameter of $1.0 \mathrm{~cm}$, internal diameter of $2.35 \mathrm{~mm}$, electrical resistance of $R=1000 \Omega$ and situated in front of the permanent magnet attached at the membrane at $0.4 \mathrm{~cm}$. The coil was fixed at the top of the aluminium frame, and aluminium was chosen as a non-magnetic material to avoid damping effects in the system. On the other hand, the permanent magnet was a strong neodymium magnet $20 \mathrm{~g}$ in weight and $1.2 \mathrm{~cm}$ in diameter with a magnetic field at its surface of $0.2 \mathrm{~T}$. Figure 8 and 9 show the real configurations of the system and the cavity, respectively.

\subsection{Discussion}


The resulting curve is shown in Figure 10 for the power per unit area of the membrane as a function of the velocity of water. It is seen that up to $30 \mathrm{~mW} / \mathrm{cm}^{2}$ could be extracted from residual water streams with velocities up to $1.7 \mathrm{~m} / \mathrm{s}$. Therefore, it will require a membrane area of approximately $35 \mathrm{~cm}^{2}$ for $1 \mathrm{~W}$ of output power. This performance for an energy harvester can be rated as medium-high performance if one considers that typical harvesters can extract up to $0.1 \mathrm{~W} \mathrm{~cm}^{2}{ }^{19}$. In addition, membranes are relatively inexpensive and can cover large areas.

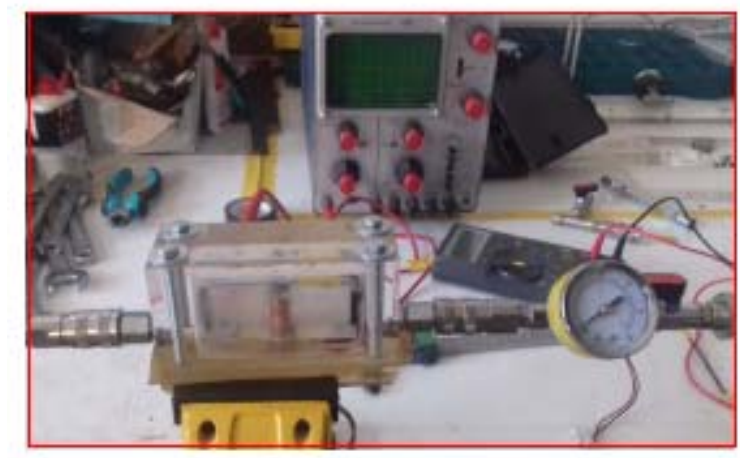

FIGURE 8 Real experimental setup.

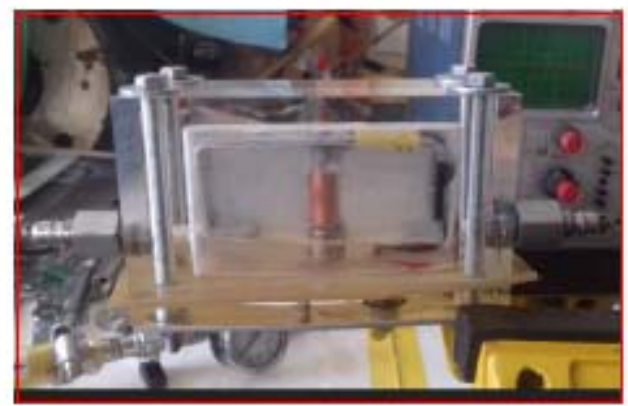

FIGURE 9 Experimental setup of the cavity.

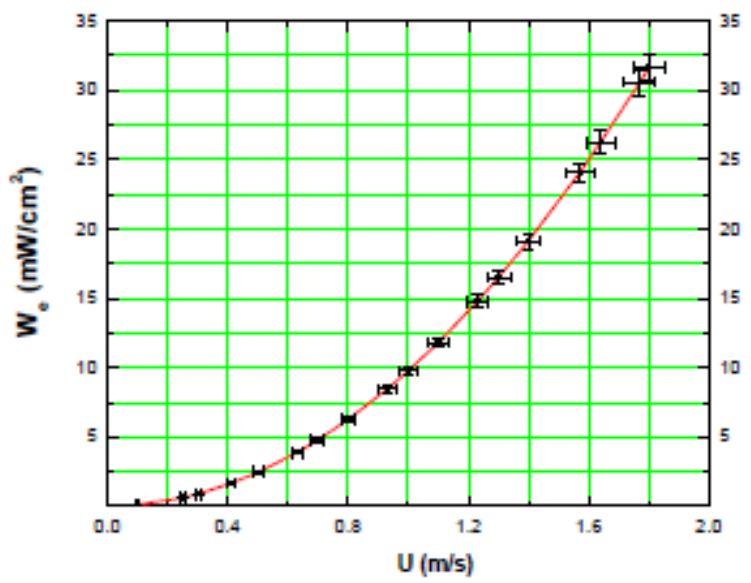

FIGURE 10 Plot of the power per unit area of the membrane as a function of the water velocity in the cavity. 


\subsection{Long-term power output stability and efficiency}

In order to assess the long-term power output stability, a series of 5 tests were performed. Each test consisted of operating the cavity at constant water flow and for 8 continuous hours and immediately followed by another period of 8 hours at rest i.e., at zero water flow and with a relaxed membrane. Therefore, the total duration of each test was of 16 hours and for the 5 tests a total of 80 continuous hours.

The resulting curve is shown in Figure 11. It is seen that the output power profile can be divided into two parts namely: The first part with a sharply increasing and decreasing of the output power followed for a second part at almost constant decrease. This is reasonably explained if one considers that initially the membrane was not stretched at its optimum position but rather a somehow higher tension, then with the normal relaxation of the membrane after certain time the membrane attains the tension which maximize its performance. Nevertheless, the relaxation of the membrane continues with time and the power is decreasing but at constant rate. This relaxation is mostly caused by the unavoidable inelastic degradation of the membrane (mostly by creep) which is caused mostly by the constant load in which the membrane is subjected by the load of the magnet.

As conclusion, it is easy to see that, in order to rectify the variation of power with time, the membrane should be stretched from time to time in order to compensate the natural creep experienced. This can be done, for example, by using a simple set of knobs or analogous system.

In the end, the life of the device will be given by the maximum elongation of the membrane which depends of the specific membrane used (material, thickness, etc.) 


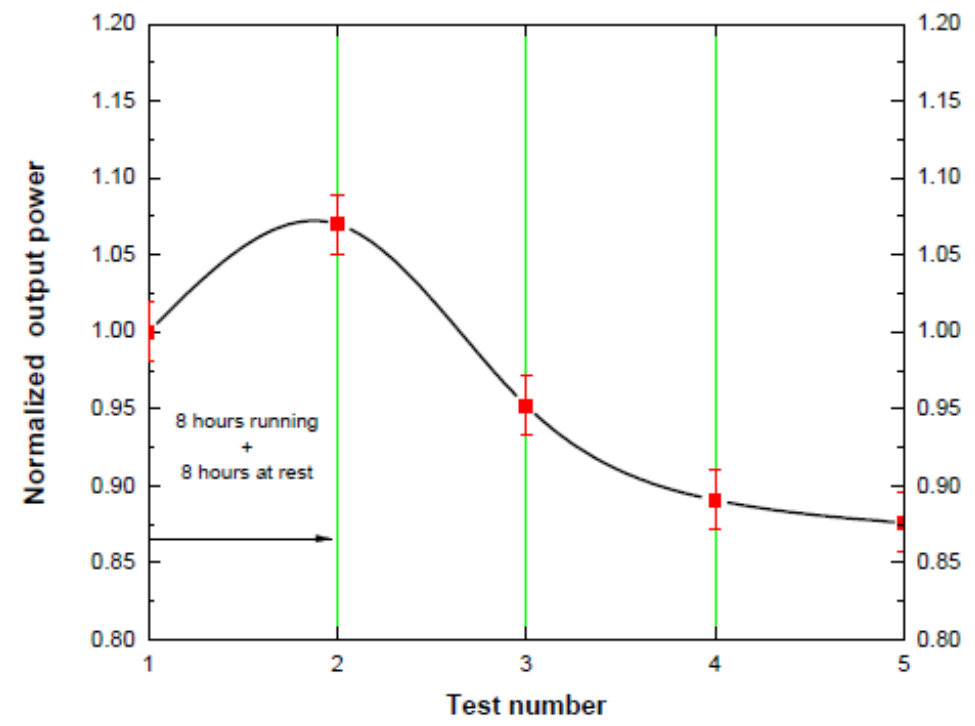

FIGURE 11. Plot of the output power profile as function of time.

\section{3 Efficiency}

The extractable upper limit of hydrokinetic power per cross sectional area from a water stream with velocity $U_{1}$ is given by

$$
w_{2}=\frac{\operatorname{sRE}}{2}
$$

where $c_{B}$ is the Betz's limit associated with duct channels and with a maximum value of $C_{D}=\frac{16}{27}=0.593$ (or 59.3\%). By dividing the density power using a membrane -given by Eq.(34), and the theoretical density power -given by Eq.(35), a power efficiency $\varepsilon_{w}$ may be defined as

$$
\begin{aligned}
& a_{W}=\frac{W_{s}}{W_{t}} \\
& =\frac{4 m_{T}^{2} \tilde{F}}{S_{R} E_{1} T}
\end{aligned}
$$

The experimental efficiency obtained is shown in Figure 12 and using a Bentz's coefficient of $c_{E}=0.593$ (or $59.3 \%$ ). It is seen that the use of membranes could be an interesting way to harness residual waters when velocities are of small magnitude which is compatible with the proposed application for this concept to residual waters as defined in preceding sections. The efficiency energy in those cases could be around 0.8 to 0.6 or thereabouts. 


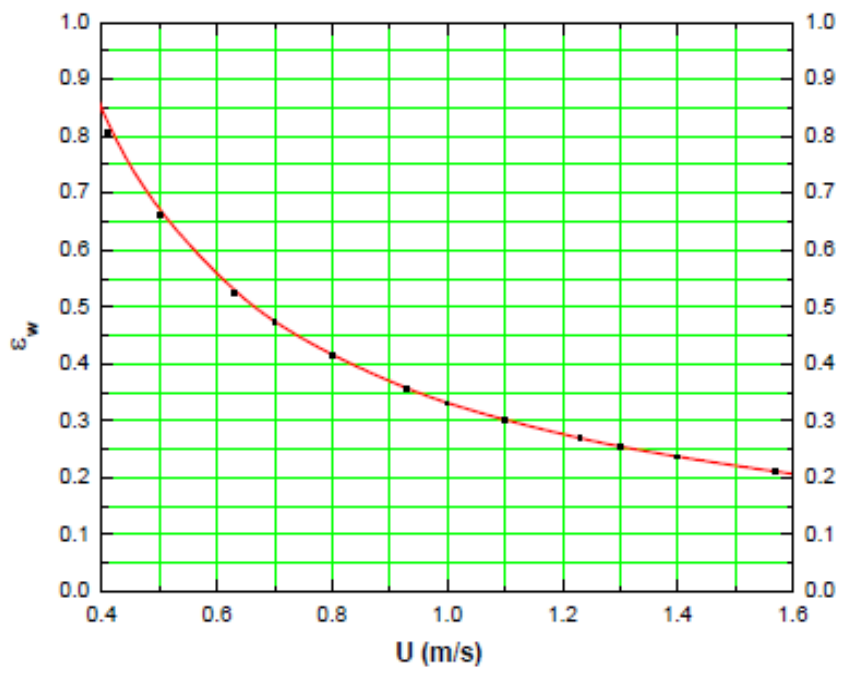

FIGURE 12. Calculated efficiency of the membrane taken from Fig 10.

\section{CONCLUDING REMARKS}

A novel hydrokinetic harvester concept has been proposed for hydrokinetic energy conversion using compliant surfaces as alternative technology for waters with a low total pressure relative to the surrounding environment that are not suitable to be turbined.

The idea is based on the bifurcation of water flow into two streams with equal static pressure but with a relative velocity owing to separation by a compliant surface (membrane). Because of Taylors instabilities from the relative velocity between the streams, steady-state harmonic motion of the membrane could be directly transformed into an output of electricity. A theoretical model was developed, and analytical expressions were derived which agreed qualitatively and quantitatively with the experiment performed. It was found that for typical residual water with a velocity of approximately $1.7 \mathrm{~m} / \mathrm{s}$, the output power is on the order of 30 $\mathrm{mW} / \mathrm{cm}^{2}$ based on the area of the membrane. Then, by a comparison with other energy harvesters, the proposed energy harvester can be rated as a high-performance energy harvester. Additional research and development is required in order to arrive at a reliable practical and commercial design.

\section{NOMENCLATURE}

$$
\begin{aligned}
& b=\text { width of the compliant surface } \\
& d=\text { differential of length } \\
& c_{1}=\text { parameter } \\
& c_{2}=\text { parameter } \\
& c_{B}=\text { Betz's coefficient }
\end{aligned}
$$




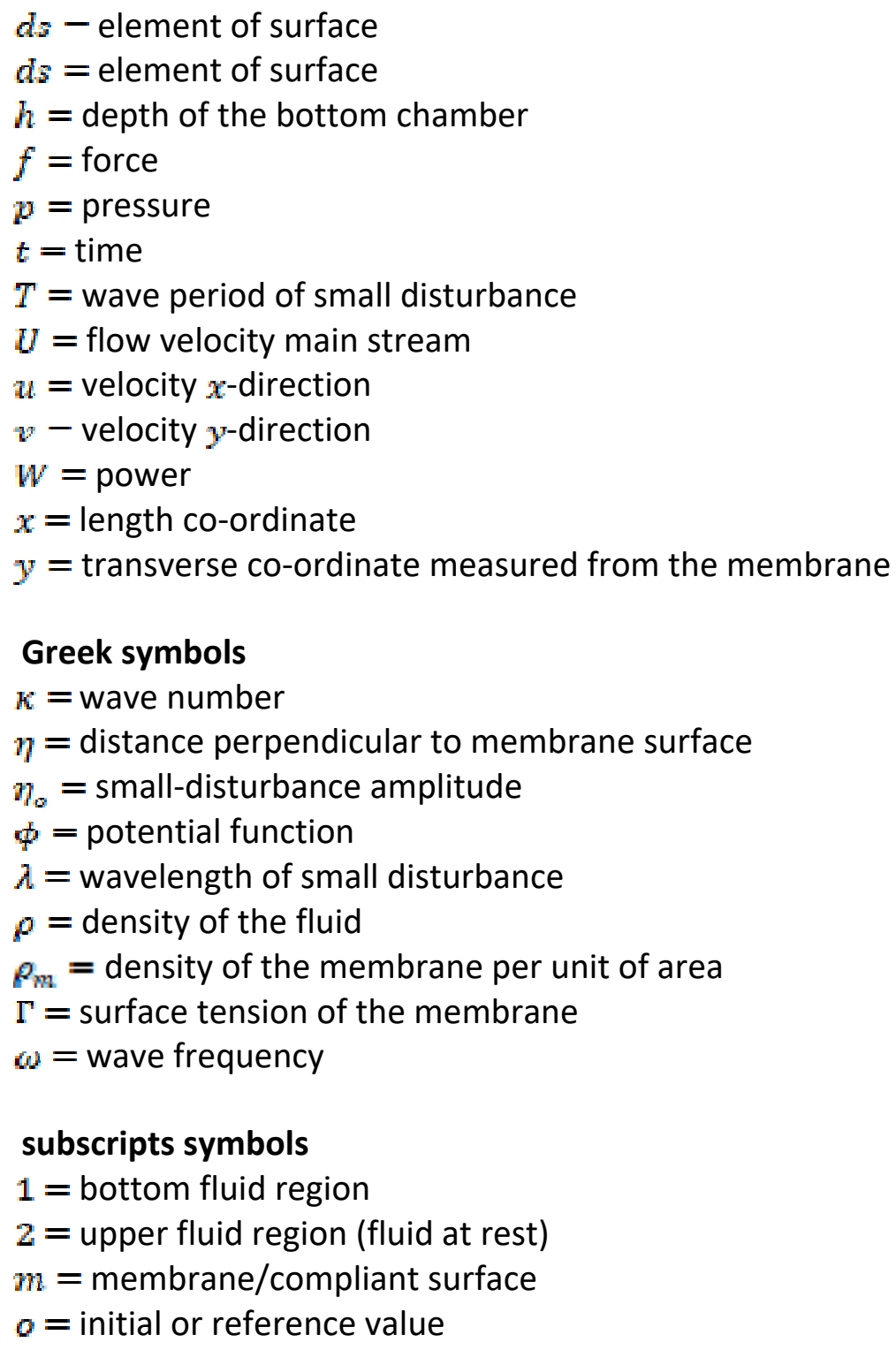

\section{ACKNOWLEDGEMENTS}

This research was supported by the Spanish Ministry of Economy and Competitiveness under fellowship grant Ramon y Cajal: RYC-2013-13459.

\section{REFERENCES}

1. Selvan KV, Hasan MN, Mohamed Ali MS. Methodological reviews and analyses on the emerging research trends and progresses of thermoelectric generators. Int J Energy Res. 2019;43:113-140. 
2. Shaislamov U, Kim Y, Kim WS, Jeong H, Lee HJ, Chun W. Hybrid operation of triboelectric nanogenerator for electricity generation by a lowtemperature differential heat engine. Int. J. Energy Res. 2017; 41: 1412-1421.

3. Al-Nimr M'd, Kiwan S, Sharadga H. A hybrid TEG/wind system using concentrated solar energy and chimney effect. Int J Energy Res. 2018;42:2548-2563.

4. Sun X, Zhang L, Huang D, Zheng Z. New insights into aerodynamic characteristics of oscillating wings and performance as wind power generator. Int J Energy Res. 2018;42:776-789.

5. Silleto MN, Yoon SJ, Arakawa K. Piezoelectric cable macro-fiber composites for use in energy harvesting, Int. J. Energy Res. 2015;39, 120-127.

6. Muhthassim B, Thian XK, Hasan KND . Energy Harvesting from Salinity Gradient. IOP Conference Series: Earth and Environmental Science, 2018; 140, conference 1

7. Elahi H, Eugen M, Gaudenzi P. A Review on Mechanisms for Piezoelectric-Based Energy Harvesters. Energies. 2018; 11(7), 1850.

8. Chen J, Wang ZL. Reviving Vibration Energy Harvesting and Self-Powered Sensing by a Triboelectric Nanogenerator. Joule. 2017. 1(3), 480-521.

9. Siang J, Lim MH, Salman LM. Review of vibration based energy harvesting technology: Mechanism and architectural approach. Int J Energy Res. 2018;42:1866-1893.

10. Laws ND, Epps BP. Hydrokinetic energy conversion: Technology, research, and outlook. Renewable and Sustainable Energy Reviews. 2016;57:1245-1259.

11. Drew B, Plummer AR, Sahinkaya MN. A review of wave energy converter technology. Proceedings of the Institution of Mechanical Engineers, Part A: Journal of Power and Energy. 2016; 223(8): 887-902.

12. Patel V, Bhat G, Eldho TI, Prabhu SV. Influence of overlap ratio and aspect ratio on the performance of Savonius hydrokinetic turbine. Int. J. Energy Res. 2017. 41: 829-844.

13. Zhao G, Su X, Cao , Su J, Liu Y. Experiments on the hydrodynamic 
performance of horizontal axis tidal current turbine and desalination of sea water. Int. J. Energy Res. 2016; 40: 600-609.

14. Shehata AS, Xiao Q, Saqr KM, Alexander D. Wells turbine for wave energy conversion: a review. Int. J. Energy Res. 2017; 41: 6-8.

15. Lopez I, Pereiras B, Castro F, Iglesias G. Performance of OWC wave energy converters: influence of turbine damping and tidal variability, Int. J. Energy Res. 2015;39, 472-483.

16. Landau LD, Lifshitz EM. Fluid Mechanics. Pergamon Press; 1959.

17. Lamb H. Hydrodynamics. Dover; 1932.

18. Kreyszig E. Advanced Engineering Mathematics. Tenth edition. John Wiley \& Sons; 2015.

19. Dostal F. New Advances in Energy Harvesting Power Conversion. Analog Dialogue, 2015;. 49(9): 1-3 